\title{
Ferromagnetic correlations in quasi-one-dimensional conducting channels
}

\author{
Boris Spivak \\ Physics Department, University of Washington, Seattle, WA 98195, USA
}

Fei Zhou

Physics Department, Princeton University, Princeton, NJ 08544, USA

\begin{abstract}
We propose a model which explains the experimental observation of spontaneous spin polarization of conducting electrons in quasi-one-dimensional AlGaAs/GaAs channels [3, 4 . We show that a ferromagnetic order is a generic property of a quasi-one- dimensional conducting channel embedded in a Wigner crystal. We also discuss gate voltage, magnetic field and temperature dependences of the channel's conductance.
\end{abstract}


Recently both theories and experiments on conductances of quasi-one dimensional conducting channels attracted a lot of attention (See for example [1 -5]). Quasi-one-dimensional confining potential has been made by applying split gates to two-dimensional electron gas in AlGaAs/GaAs heterostructures (See the insert in Fig.1). In this case electron motion is quantized in $x$ direction. If the confining potential changes adiabatically in " $\mathrm{y}$ " direction, we can neglect backscatterings of electrons incident to the channel from the two-dimensional reservoirs. For noninteracting electrons at zero temperature and in the absence of an external magnetic field $H$, the conductance of the system is quantized

$$
G=\frac{2 e^{2}}{h} k_{1}
$$

Here $k_{1}=0,1,2 \ldots$ is an integer associated with number of quantized levels in $x$ direction, which are filled with electrons. The prefactor 2 in Eq.1 reflects the spin degeneracy of electron states at $H=0$. In experiments $\mathbb{1}$ 田, the electron concentration in two-dimensional semiconductor structures was controlled by a gate voltage $V_{g}$. Eq.1 would manifest itself in the existence of plateaus in the $V_{g}$-dependence of $G\left(V_{g}\right)$ quantized in units $\frac{2 e^{2}}{h}$. When the concentration of electrons in the channel is high, or $k_{1} \gg 1$, Eq.1 is in very good agreement with experimental data [1 4]. However, at small concentration of electrons, when $k_{1} \sim 1$, experimental data [3, 4] deviate from Eq.1 dramatically. The following anomalies have been observed:

a). At small temperature $T$, in addition to plateaus at $G=\frac{2 e^{2}}{h}, 2 \frac{2 e^{2}}{h}$, the dependence $G\left(V_{g}\right)$ exhibits "smeared plateaus" at $G \sim 0.7 \frac{2 e^{2}}{h}$ and $G \sim 1.7 \frac{2 e^{2}}{h}$.

Furthermore, in the noninteracting electron picture, an external magnetic field $H$ parallel to the film lifts the spin degeneracy and each plateau splits into two plateaus quantized in units $\frac{e^{2}}{h}$. Again, this is in agreement with experiments at large $k_{1}$. However, the plateaus at $G=0.7 \frac{2 e^{2}}{h}, \frac{2 e^{2}}{h} 1.7 \frac{2 e^{2}}{h}, 2 \frac{2 e^{2}}{h}$ do not split in the external magnetic field [3, 4 . It has been noted [3] 4] that the $H$ dependence of positions of these plateaus can be explained if one assumes a ferromagnetic electron ground state in the channel at $H=0$.

b). The first and third plateau $\left(G \sim 0.7 \frac{2 e^{2}}{h}, 1,7 \frac{2 e^{2}}{h}\right)$ are more smeared than the second 
and fourth plateau $\left(G=\frac{2 e^{2}}{h}, 2 \frac{2 e^{2}}{h}\right)$.

c) The plateaus at $G=\frac{2 e^{2}}{h}, 2 \frac{2 e^{2}}{h}$ become weaker as $\mathrm{T}$ increases, while the plateaus at $G \sim 0.7 \frac{2 e^{2}}{h}, 1.7 \frac{2 e^{2}}{h}$ become stronger.

Existing theories of one-dimensional interacting electron gas predict no spin polarization in the ground state and can not explain the measured dependence $G\left(V_{g}, H, T\right)$ when the concentration of electrons in the channel is small.

In this article we propose a qualitative explanation of experimentally observed anomalous features. Consider the confining potential $V\left(x, V_{g}\right)=V_{0}\left(V_{g}\right)\left(1+\frac{x^{2}}{A^{2}\left(V_{g}\right)}\right)$ as shown in Fig.1. If the potential $V(x)$ is shallow (the value of $A$ is big) and the concentration of electrons in the channel is small, the ground state of the electron system is a Wigner crystal. In Fig.1 positions of localized electrons in the Wigner crystal are indicated by solid dots. We will assume that the Wigner crystal is pinned and the conductance of the system is determined by hoppings of point defects in the crystal (interstitial electrons or vacancies), concentration of which is exponentially small. The activation energy $E^{i}(x)$ of interstitial electrons is nonuniform and has a minimum $E_{m}^{i}\left(V_{0}\right)=E^{i}(x=0)$ in the middle of the channel.

At a critical value $V_{0}=V_{0 c}$, melting of Wigner crystal occurs near $x=0$ and a quasi-one dimensional conducting channel is formed. Below we consider two scenarios where creation of the channel corresponds to a first and second order phase transition. In both scenarios the channel is ferromagnetic.

\section{SECOND ORDER LIFSHITZ PHASE TRANSITION}

Let us assume that at the critical value $V_{0}=V_{c 0}$, for the first time, the activation energy of the interstitials becomes zero in the middle of the channel, i.e. $E_{m}^{i}\left(V_{0}=V_{0 c}\right)=0$. This can be considered as a second order Lifshitz phase transition. The interstitial electrons are indicated in Fig.1 by the symbol "*". This transition is a precursor of melting of a crystal into a liquid. The existence of zero point motion defects in the middle of the channel is a nonuniform analogy of the model of supersolid proposed by A.F.Andreev and I.M Lifshitz in 
the context of quantum melting of helium crystals [6]. Such a state has never been observed in bulk helium crystals. Latter it was noted [7] that conditions for existence of such a state are less restrictive at a crystal-liquid surface, where the amplitude of quantum fluctuations of the lattice sites is larger. This leads to a quantum rough state of the surface, where zero point motion steps on the surface exist in the ground state, which, in turn, leads to the existence of surface crystallization waves at $T=0$ [7]. Crystallization waves at small $T$, have been observed [8], while existence of solid-liquid helium quantum rough surfaces in $\mathrm{d}=3$ case is still an open question [9, 10]. In two dimensional case the existence of quantum rough surfaces is more plausible than in the three dimensional case because the amplitude of quantum fluctuations of particles at the surface is larger.

To begin, we first consider the case when all spins are polarized in $\downarrow$ direction, for example, by an external magnetic field parallel to the film. In this case the interstitial's energy spectrum in the quasi-one dimensional channel has a form

$$
\epsilon_{\downarrow}\left(k_{x}, p_{y}\right)=\epsilon_{0}\left(k_{x}+\frac{1}{2}\right)+\Delta_{\downarrow} \cos \left(a p_{y}\right) .
$$

Here $k_{x}=1,2$.. is an integer corresponding to quantization of the wave function in $x$ direction, $p_{y}$ is a momentum in $y$ direction, $\epsilon_{0}=\frac{\hbar^{2}}{2 m^{*} L^{2}}, L=\left(\frac{\hbar A}{2 \sqrt{2 e V_{0} m^{*}}}\right)^{\frac{1}{2}}$ is a characteristic dimension of the wave function in $x$ direction, $m^{*} \sim \frac{\hbar^{2}}{\Delta_{\downarrow} a^{2}}$ is the effective mass of the interstitial, $\Delta_{\downarrow}$ is the band width for interstitials with spin $\downarrow$ direction, and $a=\frac{1}{\sqrt{n}}$ and $n$ are interelectron distance and electron concentration in the Wigner crystal respectively. Band structures for interstitials moving in a polarized Wigner crystal in the case $\epsilon_{0}>\Delta_{\downarrow}$ are shown in Fig.2.

In the absence of an external magnetic field $H=0$, the interstitial electrons wave functions and spectrum inside the conducting channel depend crucially on the magnetic structure of the Wigner crystal. The magnetic structure of a uniform Wigner crystal is not known (See a discussion in [11]). We will assume that the magnetic exchange energy between electrons $J$ has an antiferromagnetic sign and the ground state corresponds to either an antiferromagnetic state or a resonating valence bonds (RVB) state [14]. We will 
show that in both cases the conducting channel should be ferromagnetic. It has been noted [12,13] that in some range of concentrations the ferromagnetic state of the Wigner crystal is also possible. In this case, our results are valid at relatively high temperatures $T>J$. We believe that in experiments [3, [4] this inequality took place because in the Wigner crystal $J \sim 1 m K[13]$.

It has been known for a long time [15 20] that a point defect embedded in a uniform antiferromagnetic quantum crystal is surrounded by a ferromagnetic region with a radius

$$
R \approx a\left(\frac{\Delta_{\downarrow}}{J}\right)^{1 / 4} \gg a
$$

We assume that $\Delta_{\downarrow} \gg J$. The reason for the existence of the magnetic polaron is that $\Delta_{\downarrow}$ is much larger than the energy band width of the defect embedded in an antiferromagnetic crystal. A competition between negative delocalization energy of the point defect inside the ferromagnetic polaron ( of order of $\frac{\hbar^{2}}{2 R^{2} m^{*}}$ ) and the positive spin polarization energy of the magnetic polaron, (of order of $\left.J\left(\frac{R}{a}\right)^{2}\right)$ leads to Eq.3.

If $T>J$ one should substitute $J$ with $T$ in Eq.3.

Thus there are two characteristic lengths in the problem, $L$ and $R$. Let us consider the case $L<R$ when the magnetic polarons have sizes $R$ and $L$ in $y$ and $x$ direction respectively. If $N$, the one-dimensional concentration of the interstitial electrons in the channel, is small $\left(N \ll R^{-1}\right.$ ), the magnetic polarons do not overlap; the magnetization $\boldsymbol{M}(y)$ is nonuniform; the channel conducts poorly and the conductance of the channel increases gradually with $N$ (or with $V_{g}$ ). At $N \gg R^{-1}$, the magnetic polarons overlap and form a conducting magnetically polarized channel. The width of the magnetically polarized region in this case is of order of $L$. At this point the concentration of interstitials in the channel is small and, due to exchange spin splitting, they have spins $\downarrow$. The conductance of the channel exhibits the first plateau at $G=\frac{e^{2}}{h}$.

The situation arising at a high concentration of interstitials $N$ depends on a ratio between $\epsilon_{0}$ and $\Delta_{\downarrow}$. If $\epsilon_{0} \gg \Delta_{\downarrow}$, further increasing of $N$ will lead to filling of the band $k=1, \uparrow$. Since $\Delta_{\uparrow}<\Delta_{\downarrow}$, the kinetic energy of interstitials with spins $\uparrow$ is small (See Fig.2). Here 
$\Delta_{\uparrow}$ is the width of the band with $k=1, \uparrow$. The width of the magnetic stripe does not increase as the band $k=1, \uparrow$ is being filled and interstitials in the band $k=1, \uparrow$ move in the uniform exchange potential associated with the uniform magnetization $\boldsymbol{M}$ inside the channel. Therefore the conductance exhibits the second plateau at $G=\frac{2 e^{2}}{h}$, which is not smeared.

At last, let us discuss the temperature dependence of the first plateau, which corresponds to the band $k=1, \downarrow$. Suppose that $\left(V_{0}-V_{0 c}\right)<0$ and $\left|V_{0}-V_{0 c}\right|$ is small and, consequently, the interstitial activation energy $E_{m}^{i}>0$ is also small. In this situation interstitials thermally activated in the channel increase the magnetic moment $\mathbf{M}$ and make the distribution of $\boldsymbol{M}(z)$ more uniform. As a result, the first plateau at $G \sim \frac{e^{2}}{h}$ becomes stronger as temperature increases. Since $E_{m}^{i}$ is small, one can neglect thermal fluctuations of the Wigner crystal lattice. This is different from solid ${ }^{3} \mathrm{He}$ case where the activation energy of point defects is large. Therefore the crystal melts before point defects contribute significantly to magnetic properties of the sample.

The presented above model explains the following experimental facts.

a).It predicts the ferromagnetic ground state of the conducting channel and the existence of an additional step in the $V_{g}$-dependence $G\left(V_{g}, \mathrm{H}=0\right)$ at $G=\frac{e^{2}}{h}$.

b).It predicts that the first plateau in the $V_{g}$-dependence of $G\left(V_{g}\right)$ is more smeared than the second one.

c).It explains why the first plateau at $G \sim \frac{e^{2}}{h}$ becomes stronger as $T$ increases.

d).It also follows from the presented above picture that at small concentration of electrons in the channel when $G \ll \frac{e^{2}}{h}$, and at small $T$, the system should exhibit giant negative magnetoresistance in a parallel magnetic field. This is because the radius of the magnetic polaron $R(H)$ increases with $H$. Obviously, this effect takes place also in the variable range hopping regime [21].

At last, let us mention difficulties of the presented above model.

1). Experimental data [3,4] exhibit the plateau at $0.7 \frac{2 e^{2}}{h}$. It is not clear to us whether it 
is possible to attribute the difference between $0.7 \frac{2 e^{2}}{h}$ and $\frac{e^{2}}{h}$ to smearing of the first plateau.

2). There are experimental indications that the first and the third plateau disappear at $T=0$ [22,23]. I To this end, we would like to mention that the filling of the first band, as $V_{g}$ changes, is a complicated process which involves overlapping of the magnetic polarons. Therefore, even in the approximation when there is no direct interaction between the interstitials, there is an indirect long range many-body effective interaction between the interstitials, which is mediated by spin degrees of freedom of the Wigner crystal. As a result, if $N$ is fixed, the spatial phase separation of the interstitials takes place at relatively small $N$ [18]. Recently this phenomenon has been reconsidered in the context of HTC [24]. The apparent differences between [24] and the situation discussed here are: a)the interstitials are neutral objects because they are screened by Wigner crystal lattice and b) in experiments it is the chemical potential rather than $N$ that is fixed. The latter means that at $T=0$ there is no phase separation. Instead, $N\left(V_{g}\right)$ dependence exhibits a jump from zero to a finite value of the interstitial concentration $N=N_{c}>0$. The final state of the conducting channel in this case is uniform. As it is usual for first order phase transitions, the states with $N=0$ and $N=N_{c}$ are separated by an energy barrier. Thus, the formation of the conducting channel is a collective phenomenon which involves tunneling of many electrons into the channel. Therefore, at this point, we can not rule out a possibility that the $G\left(V_{g}, T, H\right)$ dependence is hysteretic and that the experimental results depend on the history of the temperature, the magnetic field and the gate voltage.

3). In the approximation of noninteracting interstitials and at $\Delta_{\downarrow}<\epsilon_{0}$ the band $k=1$, $\uparrow$ will be completely filled before the the chemical potential reaches the top of the band $k=1, \downarrow$. This would lead to reentrance from the plateau at $G=2 \frac{e^{2}}{h}$ to the plateau at $G=\frac{e^{2}}{h}$ as $N$ increases.

It is worth emphasising, however, that the presented above consideration is of a single

\footnotetext{
${ }^{1}$ We are grateful to D.Khmelnitskii for turning our attention to this difficulty.
} 
particle nature. It neglects interstitial-interstitial and interstitial-Wigner crystal lattice interactions and requires detailed knowledge of interstitials band structures at relatively high interstitial energies. In experimental situation, $\frac{R}{a}=\left(\frac{\Delta_{\downarrow}}{J}\right)^{1 / 4}$ is not such a big number and the interaction effects could be very important at large $N$. In particular, the quasiparticle's bandwidth could depend on $N$.

Some of these difficulties may be resolved if we consider the opposite limit $\epsilon_{0} \ll \Delta_{\downarrow}$. In this case $k^{*} \sim \frac{\Delta_{\downarrow}-\Delta_{\uparrow}}{\epsilon_{0}}$ levels with spin $\downarrow$ should be filled before the chemical potential of interstitials reaches the band $k=1, \uparrow$. Therefore the conductance is quantized in unit $\frac{e^{2}}{h}$ and plateaus in $G\left(V_{g}\right)$ follow in a proper sequence. It is also plausible that the first plateau becomes stronger as $T$ increases. It is not clear, however, why in the framework of this picture the third plateau at $G=\frac{3 e^{2}}{h}$ should be more smeared than the second one at $\frac{2 e^{2}}{h}$.

In principle, it would be possible to distinguish $\epsilon_{0}>\Delta_{\downarrow}$ and $\epsilon_{0}<\Delta_{\downarrow}$ cases by different $V_{g}^{i}(H)$ dependences. Here $V_{g}^{i}$ are the gate voltages at which the chemical potential reaches the bottom of the $i^{\text {th }}$ band and a new plateau occurs. In the case $\Delta_{\downarrow}<\epsilon_{0}$, the gate voltages $V_{g}^{1}$ and $V_{g}^{2}$ correspond to bottoms of $k=1, \downarrow$ and $k=1, \uparrow$ bands. Therefore they move in the opposite directions as $H$ increases. In the case $\Delta_{\downarrow}>\epsilon_{0}$, gate voltages $V_{g}^{1}$ and $V_{g}^{2}$ correspond to bottoms of $k=1 \downarrow$ and $k=2 \downarrow$ bands and they move in the same direction as $H$ increases. In experiments [3,4], $V_{g}^{1}$ and $V_{g}^{2}$ move in the same direction. It is not clear, however, whether this can also be explained as an orbital magnetic field effect which exists due to a finite width of the two dimensional electron gas [3, 4 ].

\section{CONDUCTING CHANNELS WITH LARGE WIDTH}

In a uniform situation, the two-dimensional Wigner crystal melting is believed to be a first order phase transition, which occurs at a critical electron concentration $n_{c}$. In a nonuniform case shown in Fig.1, a classical picture would predict that the transition takes place at a larger concentration of electrons in the middle of the channel $n(x=0)=n_{c}^{\prime}>n_{c}$ because of existence of the crystal-liquid surface energy. The width of the conducting channel 
at the point of transition is $D_{c}=\left(\frac{3 \alpha A^{2}}{2 \gamma}\right)^{1 / 3} \gg a$. Here $\gamma=\left.n_{c} \frac{\partial\left[E_{L}(n)-E_{W}(n)\right]}{\partial n}\right|_{n=n_{c}}, E_{L}(n)$ and $E_{W}(n)$ are energy densities of 2D Fermi liquid and Wigner crystal respectively, and $\alpha$ is the surface energy per unit length.

Now consider a situation when the width $D$ is large and the concentration of electrons in the channel is high. In this case we can view the system as a stripe of Fermi liquid sandwiched between two antiferromagnetic (or RVB) Wigner crystal (See Fig.3a).

It has been pointed out in the context of solid-liquid ${ }^{3} \mathrm{He}$ interface [25] that the ferromagnetic ordering is a generic property of a quantum rough surface. It originates from the competition between kinetic energy of defects on the surface and the antiferromagnetic exchange energy in the bulk of crystal. Since it is not known whether the Wigner crystal-Fermi liquid surface is in a quantum smooth or in a quantum rough state, we will consider the case of a quantum smooth boundary, which is less favorable for the ferromagnetism, and present a model which exhibits boundary ferromagnetism even in this case.

We will model the system with the help of the Hubbard Hamiltonian defined on a semiinfinite two-dimensional square lattice shown in Fig.3a.

$$
H_{D}=t \sum_{i, j, \sigma} a_{i \sigma}^{\dagger} a_{j \sigma}+\text { c.c. }+U \sum_{i} a_{i \downarrow}^{\dagger} a_{i \downarrow} a_{i \uparrow}^{\dagger} a_{i \uparrow} .
$$

Here $i, j$ labels adjacent sites on a square lattice, $a_{i \sigma}, a_{i \sigma}^{\dagger}$ are annihilation and creation electron operators on a site $i, \sigma$ is a spin index labeling $\uparrow$ and $\downarrow$ directions of electron spins, $t$ and $U$ are parameters which describe intersite tunneling and intrasite electron repulsion respectively. In the case of half-filling and at $t \ll U$, the Hamiltonian describes a Mott insulator, which is an analog of the Wigner crystal. The spin ground state of the system is antiferromagnetic (or RVB) with exchange energy of order $J \sim \frac{t^{2}}{U}[26]$.

We will describe the semi-infinite two-dimensional Fermi-liquid with the help of the Hamiltonian for noninteracting electrons $H_{L}=\sum_{\boldsymbol{q}} \epsilon_{\boldsymbol{q}} b_{\boldsymbol{q}}^{\dagger} b_{\boldsymbol{q}}$, where $b_{\boldsymbol{q}}, b_{\boldsymbol{q}}^{\dagger}$ are annihilation and creation electron operators in the liquid, $\epsilon_{\boldsymbol{q}}=\frac{\boldsymbol{q}^{2}}{2 m}-E_{F}$ is the electron energy, $\boldsymbol{q}$ and $m$ are the momentum and mass respectively, $E_{F}$ is the liquid's Fermi energy. The tunneling between the Mott insulator and the Fermi liquid is described by the Hamiltonian 


$$
H_{T}=t_{1} \sum_{i^{\prime}, \boldsymbol{q}} a_{i} b_{\boldsymbol{q}}^{\dagger}+c c .
$$

Here index $i^{\prime}$ labels the localized states in the insulator at the boundary. The total Hamiltonian is $H=H_{D}+H_{L}+H_{T}$. In the case of half filling, the chemical potential of the Mott insulator lies in the middle of the gap between the valence and the conduction band (See Fig.3b). For a Wigner crystal, it means that the energy of creation of a vacancy and an interstitial electron would be the same. In general, there is no reason to expect such a symmetry in a real Wigner crystal. Therefore we introduce into the model a small concentration of impurity levels, which pin the position of the chemical potential below (or above) the middle of the gap. These impurities are marked by symbols "+" in Fig.3b. The distance between the impurities will be much larger than all other characteristic lengths in the problem. To be concrete we consider the situation when the chemical potential is pinned near the bottom of the valence band and the energy for transferring an electron into the liquid and creation of a vacancy in the Mott insulator is $U_{1} \ll U$ (See Fig.3b).

The tunneling between the liquid and the Mott insulator leads to a negative correction to the ground state energy of the system. The value of the correction depends on the spin configuration in the Mott insulator. In the second order perturbation theory with respect to $t_{1}$, we have an expression for the correction to the energy per unit surface length

$$
\delta E^{t_{1}}=-\frac{a^{3}}{L_{x} L_{y} D} \sum_{\mathbf{q}, \mathbf{n}} \frac{t_{1}^{2}}{U_{1}+\epsilon_{\{S\}}(\mathbf{n})+\epsilon_{\mathbf{q}}}
$$

where $a$ is the lattice spacing, $\epsilon_{\{S\}}(\mathbf{n})$ is the energy of a vacancy moving in a spin configuration $\{S\}$ in Mott insulator, $\mathbf{n}$ labels the vacancy eigenfunctions in the Mott insulator and $L_{y}$ is the sample length in y direction. Suppose that in the Mott insulator near the surface there is a ferromagnetic region of the width $L_{x}$ (See Fig.3a). Then we can estimate $\epsilon_{\{S\}}(\mathbf{n}) \sim \Delta \cos \left(p_{y} a\right)+k_{x}^{2} \frac{\hbar^{2}}{2 m^{*} L_{x}^{2}}$. Here $\Delta=2 t$ is the vacancy energy bandwidth in a completely spin polarized Mott insulator, $k_{x}$ is an integer corresponding to quantized motion of the vacancy in $x$-direction, $p_{y}$ is the quasi-momentum of the vacancy in " $y$ " direction $\left(\mathbf{n}=k_{x}, p_{y}\right)$. We assume that in the case of the antiferromagnetic ordering the bandwidth of the vacancy is small and it is almost localized. 
Expanding Eq.6 with respect to $\frac{t_{1}}{U_{1}} \ll 1$ and taking into account the energy of the spin polarization in the stripe of the width $L_{x}$, we get an estimate for spin configuration dependent part of the ground state energy

$$
\delta E_{\{S\}} \sim-\frac{t_{1}^{2} t^{2}}{E_{F} U_{1}^{2} L_{x}}+\frac{J L_{x}}{a^{2}} .
$$

Eq.7 has a minimum at

$$
L_{x}=a \sqrt{\frac{t_{1}^{2} U}{U_{1}^{2} E_{F}}} .
$$

Therefore the system is magnetically polarized at the surface provided $\frac{t_{1}^{2} U}{U_{1}^{2} E_{F}} \gg 1$.

The statement about existence of the magnetic polarization near the surface can be proved in a more rigorous way starting with usual perturbation theory

$$
\Delta E^{t_{1}}=\frac{t_{1}^{2}}{\epsilon_{F}} \sum_{i, i^{\prime}, \Gamma\left(i, i^{\prime}\right)} \int d \epsilon \frac{C\left(n_{\Gamma}\right)}{\epsilon+U_{1}}\left(\frac{t}{\epsilon+U_{1}}\right)^{n_{\Gamma}} J_{1}\left(i, i^{\prime}\right) .
$$

Here $i, i^{\prime}$ labels the lattice sites at the boundary, $\Gamma\left(i, i^{\prime}\right)$ labels paths of a vacancy on the lattice intersecting the boundary at sites $i$ and $i^{\prime}$, which preserve an initial spin configuration of the system ( An example of such a path is shown in Fig.3a), $J_{1}\left(i, i^{\prime}\right.$ ) is a function of the distance between sites $i$ and $i^{\prime}$, which reflects the Friedel oscillations in the Fermi liquid, $n_{\Gamma}$ is the number of steps a vacancy hops along a path $\Gamma$. While obtaining Eq.9 from Eqs.4,5, we neglected all terms which involve double electron occupancies of lattice sites. The factor $C\left(n_{\Gamma}\right)$ depends on the spin configuration in the system. In the ferromagnetic case, due to Fermi statistics of electrons, it is equal to $(-1)^{n_{\Gamma}}$. In the limit $p_{F} a \gg 1, J_{1}=\delta_{i, i^{\prime}}$ and we only need to take into account paths which enter and leave the Mott insulator through the same lattice site $i=i^{\prime}$. At this point the problem is reduced to that considered in 15, 16, where it was proven that the ground state of the system with one vacancy and infinite $U$ is ferromagnetic. A resummation of these ferromagnetic paths leads to Eq.6.

If the surface is in a quantum rough state the ferromagnetic ordering at the surface is even more favorable energetically [25]. 
The presented above picture is relevant when the width $D$ of the Fermi liquid stripe is large, which corresponds to large $k_{1}$ in Eq.1. Strictly speaking, in this case, each plateau with given $k_{1}$ also splits into two plateaus quantized in unit $\frac{e^{2}}{h}$. However, the amplitude of this splitting of plateaus should be small.

Let us consider what happens as concentration of electrons in the channel and, consequently, $D$ decreases. It has been mentioned above that the mean field theory, which neglects quantum fluctuations of the position of the crystal-Fermi liquid boundary, predicts the creation of the conducting channel as a first order phase transition. The question arises whether quantum fluctuations of electron positions in the Wigner crystal can make this transition to be a second order one. The amplitude of quantum fluctuations of the position of the crystal-liquid boundary depends on whether the surface is in a quantum rough or a quantum smooth state. If it is in a quantum rough state, we can describe deviations of the boundary from its equilibrium classical position by a variable $X(y, t)$ and use the following effective action

$$
S=\int d y d t\left[\beta\left(\frac{d X}{d t}\right)^{2}+\alpha\left(\frac{d X}{d y}\right)^{2}+\tilde{V}(X)\right]
$$

to estimate the amplitude of quantum fluctuations of the position of the boundary. Here $\tilde{V} \sim \xi X^{2}$ corresponds to the energy difference between the solid and liquid phase as the boundary is moved by distance $X, \xi=\gamma \frac{D_{c}}{A^{2}}$. The second term in Eq.10 corresponds to the surface tension and the first term corresponds to acceleration of steps along the surface.

We would like to mention that the situation described by Eq.10 is different from that considered in [7]. The electron density profile $n(x)$ in the channel is determined by the long range Coulomb force. Therefore, in our case there is no redistribution of the electron density in the liquid associated with oscillations $X(y, t)$. Using Eq.10 we get an estimate < $\left.(\delta X)^{2}\right)>\sim \frac{1}{\sqrt{\alpha \beta}} \log \frac{\alpha}{\xi a}$. Here the brackets $<>$ stand for the quantum mechanical averaging. If $\left\langle(\delta X)^{2}\right)>\gg D_{c}^{2}$, then the one-dimensional melting can occur as a second order phase transition and we arrive at the picture considered in the previous section. We believe that, in principle, this inequality can take place because the transition occurs at $r_{s} \sim 39$ where 
at short distances the crystal differs from the liquid very little.

In conclusion we would like to make the following remarks. At finite temperature the ferromagnetic ordering of the quasi-one dimensional stripe will be destroyed by thermal fluctuations. Strictly speaking, at $T=0$, the presented above arguments only prove existence of strong ferromagnetic correlations at "small" distances. At this stage we can not rule out a possibility that at "large" distances the ferromagnetism will be destroyed by quantum fluctuations of spin configuration in a 2-D Wigner crystal. Quantization of the conductance

of the channel in units $\frac{e^{2}}{h}$, however, persists as long as the length of the channel is shorter than the characteristic distance of the destruction of the ferromagnetism.

It has been shown [27] that the ground state of strictly one-dimensional systems can not be ferromagnetic. However, in the experiment [3, 4] the system is not strictly onedimensional. We are unaware of any theorem which prevents existence of ferromagnetism in quasi-one-dimensional system of the type shown in Fig.3. We leave this questions for future investigation.

We are grateful to C. Markus, D. Thouless, S. Kivelson, D. Khmelnitskii, E. Lieb and M. den Nijs for useful discussions. Work of B.S. is supported by Division of Material Sciences, U.S.National Science Foundation under Contract No.DMR-9625370. F.Z. is supported by ARO under DAAG 55-98-1-0270. F.Z. also likes to thank NECI in Princeton for its hospitality. 


\section{REFERENCES}

[1] T. J. Thornton et.al., Phys. Rev. Lett. 56, 1198(1986).

[2] B. J. van Wees, et.al., Phys. Rev. Lett. 60, 848(1988).

[3] K. J. Thomas, J. T. Nicholls, M. Y. Simmons, M. Pepper, D. R. Mace and D. A. Ritchie, Phys. Rev. Lett. 77, 135(1996);

[4] K. J. Thomas, J.T.Nicholls, M.J.Appeyard, M.Y.Simmons, M.Pepper, D.R.Mace, W.R.Tribe, D.A. Ritchie, Phys.Rev. B58, 4846(1998).

[5] C.W.J.Beenakker, H.van Houten, in Solid State Physics, edited by H.Ehrenreich and D.Turnbull (Academic Press, NY, 1991).

[6] A. Andreev, I. Lifshitz, Sov.Phys. JETP 29, 1107(1969)[Zh. Eksp. Teor. Fiz. 56, 2057(1969)].

[7] A. F. Andreev, A. Ya. Parshin, Sov. Phys. JETP 48, 763(1978)[Zh. Eksp. Teor. Fiz. $75,1511(1978)]$

[8] K. O. Keshishev, A. Ya. Parshin and A. V. Babkin, Sov. Phys. JETP 53, 500(1981) [Zh. Eksp. Teor. Fiz. 80, 716(1981)].

[9] D. S. Fisher, J. Weeks, Phys. Rev. Lett. 50, 1077 (1983).

[10] S.V.Iordansky, S.E.Korshunov, J.Low.Temp.Phys. 58 425(1985).

[11] S. Chakrovarty, S. Kivelson, C. Nayak and K. Voelker, cond-mat/9805383.

[12] C. Herring, Rev. Mod. Phys. 34, 631(1962).

[13] M. Roger, Phys. Rev. B 30, 6432(1984).

[14] P.W. Anderson, Mater. Res. Bull.,8, 153(1973).

[15] Y. Nagaoka, Phys.Rev.147, 392 (1966); Solid State Commun. 3, 409(1965). 
[16] D. J. Thouless, Proc. Phys. Soc. (London) 86, 893(1965).

[17] E. L. Nagaev, Sov. Phys. JETP 27, 122(1968) [Zh. Eksp. Teor. Fiz. 54, 228(1968)].

[18] E.L.Nagaev, "Physics of magnetic semiconductors", Nauka, Moskow, (1979).

[19] W. F. Brinkman, T. M. Rice, Phys. Rev. B2,1324(1970).

[20] A. F. Andreev, JETP Lett.24, 564(1976)[Pisma Zh. Eksp. Teor. Fiz. 24, 608(1976)].

[21] B.Spivak, Sov. Phys. JETP 60, 787 (1984).

[22] A. Kristensen, et. al., cond-mat/9808007.

[23] A.Kristensen, et. al., Physica B: Condensed Matter 249-251, 180 (1998).

[24] S. A. Kivelson, E. Fradkin and V. J. Emery, Nature 393, 550(1998).

[25] M. A. Meierovich, B. Z. Spivak, JETP Lett. 34, 551(1981)[Pisma Zh. Eksp. Teor. Fiz. 34, 575(1981)]

[26] N. F. Mott, Metal-insulator transitions, Taylor and Francis, London(1974).

[27] E. Lieb, D. Mattis, Phys. Rev. B125, 164(1962). 


\section{FIGURES}

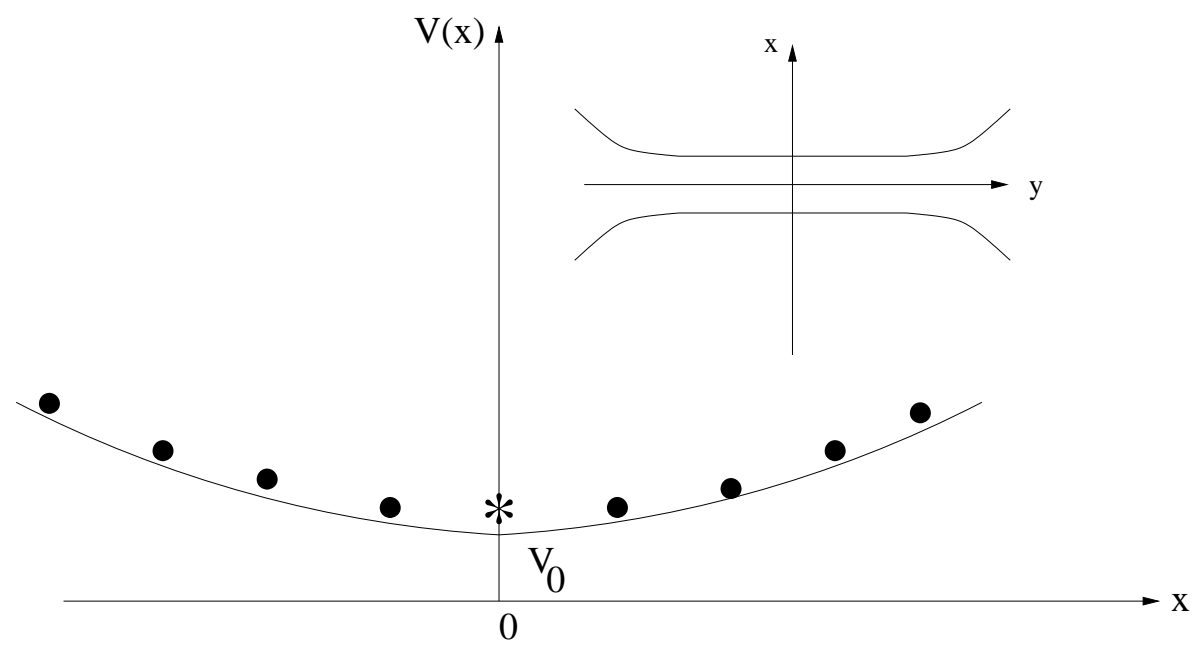

FIG. 1. The qualitative picture of the confining potential $V(x)$. Solid dots correspond to positions of electrons in the Wigner crystal. The symbol "*" corresponds to an interstitial electron. The insert shows the geometry of the conducting channel. 


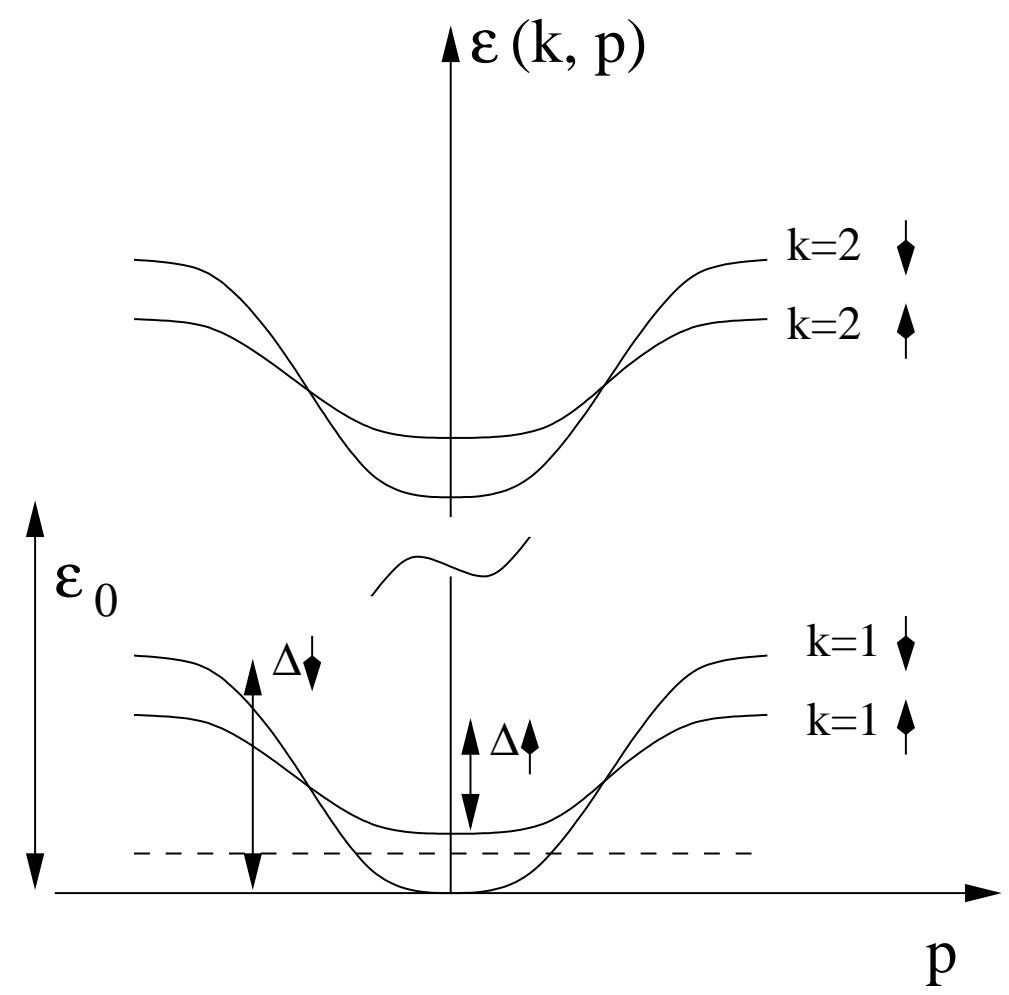

FIG. 2. A schematical picture of band structures of interstitial electrons in the case $\epsilon_{0} \gg \Delta_{\downarrow}$. The dashed line indicates the chemical potential when the channel becomes conducting. 


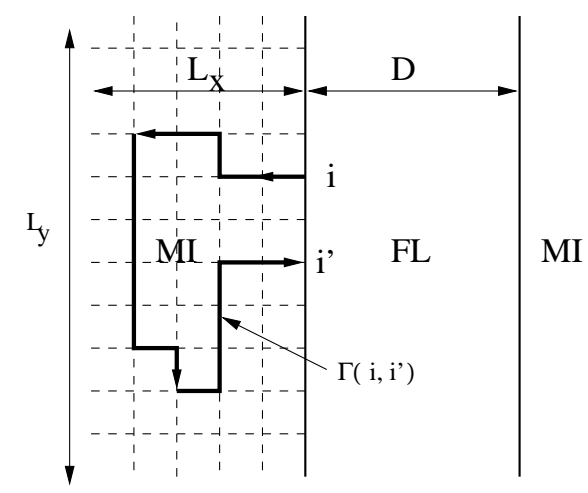

(a)

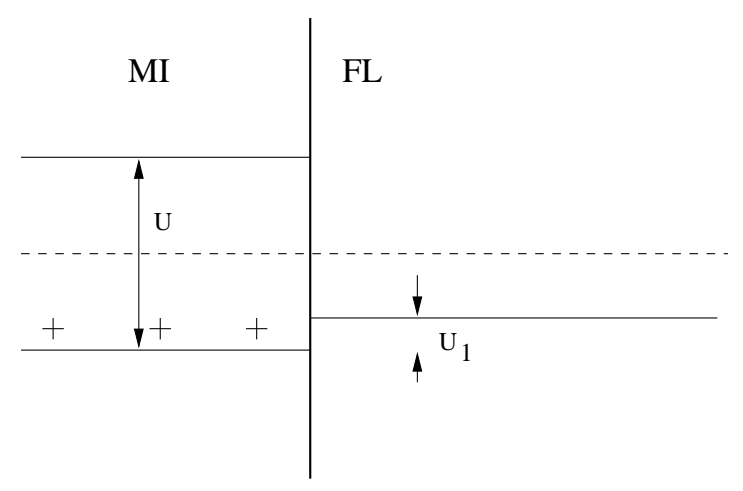

(b)

FIG. 3. a. The geometry of the Mott insulator (MI)-Fermi liquid (FL)-Mott insulator structure. The Mott insulator and the Fermi liquid are indicated by symbols "MI" and "FL" respectively. $\Gamma$ represents a path corresponding to a term in the perturbation sequence in Eq.9. b. Energy diagram for the Mott insulator-Fermi liquid boundary. 